\title{
Erdheim-Chester Disease: Case Report with Aggressive Multisystem Manifestations and Review of the Literature
}

\author{
Sultan Alotaibi ${ }^{a} \quad$ Osama Alhafi $^{a} \quad$ Hatem Nasr $^{b} \quad$ Khalid Eltayeb $^{a}$ \\ Ghaleb Elyamany ${ }^{c}$ \\ ${ }^{a}$ Department of Adult Hematology, Prince Sultan Military Medical City, Riyadh, \\ Saudi Arabia; ${ }^{b}$ Department of Radiology, Nuclear Medicine Unit, Prince Sultan Military \\ Medical City, Riyadh, Saudi Arabia; ' Department of Central Military Laboratory and \\ Blood Bank, Prince Sultan Military Medical City, Riyadh, Saudi Arabia
}

\section{Keywords}

Erdheim-Chester disease · Histiocytosis · Interferon alpha

\begin{abstract}
Erdheim-Chester disease (ECD) is an extremely rare and aggressive form of non-Langerhans cell histiocytosis. ECD usually presents with bone pain in adults aged 40-60. Its etiology is unknown but it is thought to be either a reactive or neoplastic disorder. Recently, mutation of the proto-oncogene BRAF (BRAFV600E) has been found in more than $50 \%$ of cases. The multisystemic form of ECD is associated with significant morbidity, which may arise due to histiocytic infiltration of critical organ systems. The common sites of involvement are the skeleton, central nervous system, cardiovascular system, lungs, retroperitoneum, and skin. Current available treatment is interferon alpha as the first line of treatment. Treatment with other agents is based on anecdotal case reports. Cladribine, anakinra, and vemurafenib (BRAF inhibitor) are currently advocated as promising second-line treatments for patients whose response to interferon alpha is unsatisfactory. Herein, we are reporting a middle-aged Saudi male patient with an aggressive type of ECD and highlighting the clinical, radiological, and pathological manifestations associated with ECD and the various treatment options and patient follow-up.

(C) 2017 The Author(s)

Published by S. Karger AG, Basel
\end{abstract}

\section{KARGER}

Ghaleb Elyamany, MD, PhD, FRCPC

Department of Central Military Laboratory and Blood Bank

Prince Sultan Military Medical City

Riyadh 11159 (Saudi Arabia)

E-Mail ghalebelyamany@psmmc.med.sa 


\section{Case Reports in Oncology}

\section{Introduction}

Erdheim-Chester disease (ECD) is a rare non-Langerhans cell, lipid-laden histiocytosis with specific histological and radiological findings. It was first reported by Jakob Erdheim and William Chester in 1930 [1]. ECD prevalence is unknown; roughly 550 cases have been described in the literature. The disease usually becomes apparent in adulthood between 40 and 60 years with an average age of onset of 53 years [2]. Its etiology is unknown but it is thought to be either a reactive or neoplastic disorder. However, recent detection of a mutation in the BRAF proto-oncogene has been identified in the majority of ECD patients. This recent identification of the clonal nature of the disorder has changed our understanding of the pathogenesis of the disease [2].

The diagnosis of ECD depends on the combination of clinical presentations and imaging features, which are confirmed with histopathologic findings. Skeletal involvement is highly pronounced and it occurs in up to $95 \%$ of ECD patients, and bone pain is the most common presenting symptom $[3,4]$.

Other extraskeletal involvement includes infiltration of the eyes causing exophthalmos, papilledema, xanthelasmas and papulonodular skin lesions, endocrinal infiltration and diabetes insipidus, severe lung disease, renal failure, retroperitoneal region involvement, cardiomyopathy, and central nervous system (CNS) disorders [2, 4].

The differential diagnosis of ECD includes many conditions presenting with symptoms and findings similar to ECD such as Langerhans cell histiocytosis, Rosai-Dorfman disease, Wegener's granulomatosis, multiple sclerosis, Paget disease, neurosarcoidosis, amyloidosis, metabolic disorders such as Gaucher's disease and mucopolysaccharidoses, cerebrotendinous xanthomatosis, Whipple's disease, chronic recurrent multifocal osteomyelitis, Takayasu arteritis, primary hypophysitis, and mycobacterial infections $[2,3,5]$.

Current available treatment is interferon alfa (IFN- $\alpha$ ) as the first line of treatment. Pegylated IFN- $\alpha$ is an alternative to standard IFN- $\alpha$. Treatment with other agents is based on anecdotal case reports. Agents such as cladribine, which is a new purine nucleoside analogue, is useful treatment in moderate to severe cases. Anakinra and vemurafenib (BRAF inhibitors) are currently advocated as promising second-line treatments for patients whose response to IFN- $\alpha$ is unsatisfactory $[2,6]$.

Corticosteroids may reduce the edema and exophthalmos or mild skin manifestation but are not effective in long-term monotherapy. Imatinib is a second-line treatment for patients who have abundant expression of platelet-derived growth factor receptor-beta since the presence of this mutation is suggestion a myeloid-origin disease. ECD prognosis is poor mainly in those with CNS involvement; however, after the IFN- $\alpha$ treatments, the survival increases considerably, and 5-year survival is 68\% [2].

In this paper, we present an aggressive form of ECD and discuss this rare entity including its clinical course, diagnostic challenge, treatment options and response of our patient to first-line treatment, and disease status after 1 year from the first dose. To the best of our knowledge, this is the third case from Saudi Arabia [7, 8].

\section{Case Report}

A 36-year-old male with no relevant past medical history initially presented with chronic diffused bony pain mainly around the knee and elbow joints with on and off generalized body pruritus. These complaints had been present for the last 4 years with worsening re- 


\section{Case Reports in Oncology}

ported in the previous 2 months and developed blurred vision and exophthalmos with difficulties in walking.

The patient had been investigated in multiple hospitals with different subspecialties with no definite diagnosis. The patient received analgesic and vitamin $\mathrm{D}$ for bone pain, topical light steroid creams and intravenous antihistamine injections for generalized body pruritus after each flare-up.

On admission to our department (2016), the patient's general appearance was normal and hemodynamically stable. The initial laboratory workup revealed a normal WBC count and differential, normal hemoglobin and mild thrombocytosis $\left(120 \times 10^{9} / \mathrm{L}\right)$. Renal and liver functions were normal. Tumor markers and autoimmune and rheumatologic screening were negative for all serologic markers. The inflammatory markers such as C-reactive protein (CRP) and the erythrocyte sedimentation rate (ESR) were mildly elevated.

The patient underwent radiological studies, with bilateral X-ray of the knees demonstrating bilateral sclerotic changes in the femoral and tibial bones. Humeral X-ray showed intramedullary sclerosis at the distal end of humeral bone. The lateral view showed involvement of the proximal ulnar and radial bones without pathological fracture (Fig. 1). 99mTc-methylene diphosphonate and $18 \mathrm{~F}$-fluorodeoxyglucose positron emission tomography (FDG-PET) showed bilateral symmetric uptake in the long bones, right clavicle, and mandible as well as subcutaneous and muscle soft tissue involvement in the lower limbs. Computed tomography (CT) and MRI for the lower limbs revealed multifocal intramedullary sclerosis affecting both legs and distal portions of both femoral bones. Pituitary involvement and retrobulbar soft tissue infiltration were demonstrated as enhancing lesions on MRI and high FDG uptake on PET/CT brain images. Fused PET/CT images and CT images demonstrated bilateral perinephric soft tissue thickening and uptake (Fig. 2).

Accordingly, CT-guided left proximal tibia bone biopsy was performed. Histological findings of the bone biopsy reported as diffuse infiltration by foamy histiocytes and fibrosis and rare Touton-like giant cells with immunohistochemical (IHC) profile consistent with ECD with no granuloma and negative for malignancy. On IHC staining, the histiocytes were positive for CD68, CD163, and factor XIIIa, and negative for CD1a, S100, and langerin (Fig. 3).

Once the diagnoses of ECD was confirmed and because of its aggressive nature with involvement of the orbit disturbing the patient's visual function with marked bilateral exophthalmos and gradual loss of vision, the patient underwent decompression surgery to alleviate the tissue pressure over the optic nerve, which resulted in improvement of the visual acuity.

The treatment started with pegylated IFN- $\alpha 180 \mu \mathrm{g} /$ week subcutaneously. Treatment was well tolerated and particularly efficacious for bone pain and constitutional symptoms without adverse effect and the compliance of the patient was excellent. The pain started to decline with time till disappearance by the second month of treatment, platelet count returned to normal range, and ESR and CRP deceased but were still elevated; however, they were less than the prior treatment level. The patient did not require analgesia as before the treatment.

FDG-PET and CT scan were performed after 6 months of the beginning of treatment and showed stabilization or improvement of the intraorbital and suprasellar enhancing lesions and other radiological findings detected at diagnosis which were considered as partial good response as disease stabilization was achieved and the patient's clinical status was improved. 
 Oncology}

Alotaibi et al.: Erdheim-Chester Disease: Case Report with Aggressive Multisystem Manifestations and Review of the Literature

\section{Discussion}

ECD is a rare incurable disease till date with multisystem involvement with some infiltrative predominance in the bone of the lower and upper extremities, orbit, and CNS, and the disease is aggressively related to the site of infiltration. The etiology of ECD is unknown but it is thought to be either a reactive or neoplastic disorder. Recent findings of mutations in the BRAF proto-oncogene in $>50 \%$ of ECD cases clearly add further complexity to the pathophysiology of ECD $[2,9]$.

The diagnosis is usually challenging due to the rarity of the disease and clinical overlapping with many other conditions. The diagnosis in our case study was made based on characteristic features including clinical pictures of skeletal pain, skin lesions, and radiological findings including bilateral symmetric increased tracer uptake on ${ }^{99 \mathrm{~m} T c}$ bone scintigraphy affecting the periarticular regions of the long bones, in addition to bone X-rays displaying bilateral and symmetric cortical osteosclerosis of the long bones. The definitive diagnosis was confirmed by the histological findings of infiltration of tissues with histiocytes and fibrosis and IHC staining of histocytes positive for CD68, CD163, and factor XIIIa, and negative for CD1a, S100, and langerin.

Our case presented after 4 years of complaining of bone pain. The rarity and variable presentation of this disease usually leads to delayed diagnosis and to high morbidity and mortality rates from associated complications [8].

Our patient had the classical presentation with skeletal pain and bony infiltration site and pattern and orbital involvement affecting the eye function, and his disease required urgent intervention and was controlled with first-line, pegylated IFN- $\alpha 180 \mu \mathrm{g} /$ week subcutaneously. The effective treatment options are limited with a growing promise with BRAF inhibitor, and the goal of treatment for the time being is to prolong the life expectancy and maintain a high quality of life $[2,3]$.

\section{Statement of Ethics}

Informed consent was obtained and the case study has been approved by the department's committee on human research.

\section{Disclosure Statement}

The authors have no funding or conflicts of interest to disclose.

\section{References}

1 Chester W: Lipoidgranulomatose. Virchows Arch Pathol Anat 1930;279:561-602.

-2 Diamond EL, Dagna L, Hyman DM, et al: Consensus guidelines for the diagnosis and clinical management of Erdheim-Chester disease. Blood 2014;124:483-492.

-3 Adawi M, Bisharat B, Bowirrat A: Erdheim-Chester disease (ECD): Case report, clinical and basic investigations, and review of literature. Dora Z (ed). Medicine 2016;95:e5167.

-4 Abdellateef EE, Abdelhai AR, Gawish HH, Abdulmonaem GA, Abdelbary EH, Ahmed AI: The first reported case of Erdheim-Chester disease in Egypt with bilateral exophthalmos, loss of vision, and multi-organ involvement in a young woman. Am J Case Rep 2016;17:360-370.

5 Gupta A, Kelly B, McGuigan JE: Erdheim-Chester disease with prominent pericardial involvement: clinical, radiologic, and histologic findings. Am J Med Sci 2002;324:96-100. 


\section{Case Reports in Oncology}

\begin{tabular}{l|l}
\hline Case Rep Oncol 2017;10:501-507 \\
\hline DOI: 10.1159/000477336 & $\begin{array}{l}\text { (c) 2017 The Author(s). Published by S. Karger AG, Basel } \\
\text { www.karger.com/cro }\end{array}$ \\
\hline
\end{tabular}

Alotaibi et al.: Erdheim-Chester Disease: Case Report with Aggressive Multisystem Manifestations and Review of the Literature

-6 Girschikofsky M, Arico M, Castillo D, et al: Management of adult patients with Langerhans cell histiocytosis: recommendations from an expert panel on behalf of Euro-Histio-Net. Orphanet J Rare Dis 2013;8:72.

7 Bohlega S, Alwatban J, Tulbah A, Bakheet SM, Powe J: Cerebral manifestation of Erdheim-Chester disease: clinical and radiologic findings. Neurology 1997;49:1702-1705.

-8 Binyousef RF, Al-Gahmi AM, Khan ZR, Rawah E: A rare case of Erdheim-Chester disease in the breast. Ann Saudi Med 2017;37:79-83.

-9 Haroche J, Charlotte F, Arnaud L, von Deimling A, Hélias-Rodzewicz Z, Hervier B, Cohen-Aubart F, Launay D, Lesot A, Mokhtari K, Canioni D, Galmiche L, Rose C, Schmalzing M, Croockewit S, Kambouchner M, Copin MC, Fraitag S, Sahm F, Brousse N, Amoura Z, Donadieu J, Emile JF: High prevalence of BRAF V600E mutations in Erdheim-Chester disease but not in other non-Langerhans cell histiocytoses. Blood 2012;120:2700-2703.
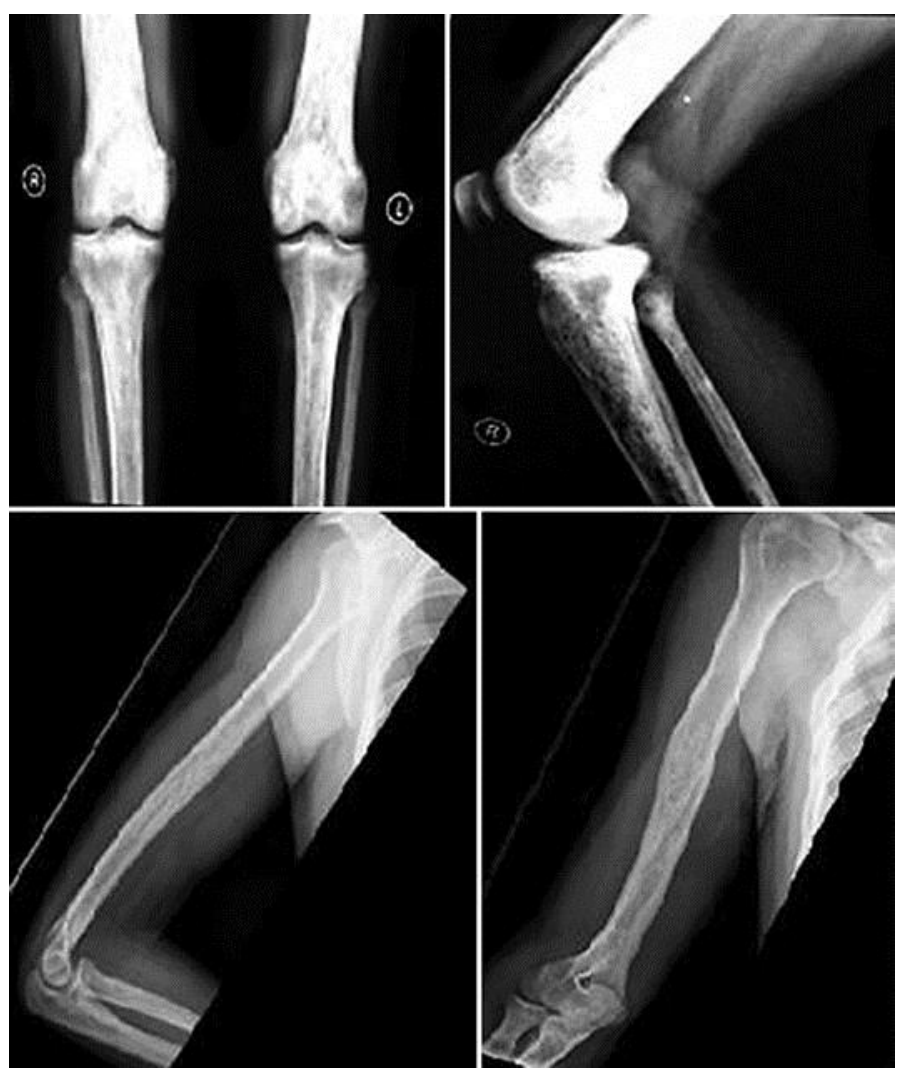

Fig. 1. Bilateral X-ray of the knees demonstrating bilateral sclerotic changes in the femoral and tibial bones. Humeral X-ray shows intramedullary sclerosis at the distal end of humeral bone. The lateral view shows involvement of the proximal ulnar and radial bones without pathological fracture. 


\section{Case Reports in Oncology}
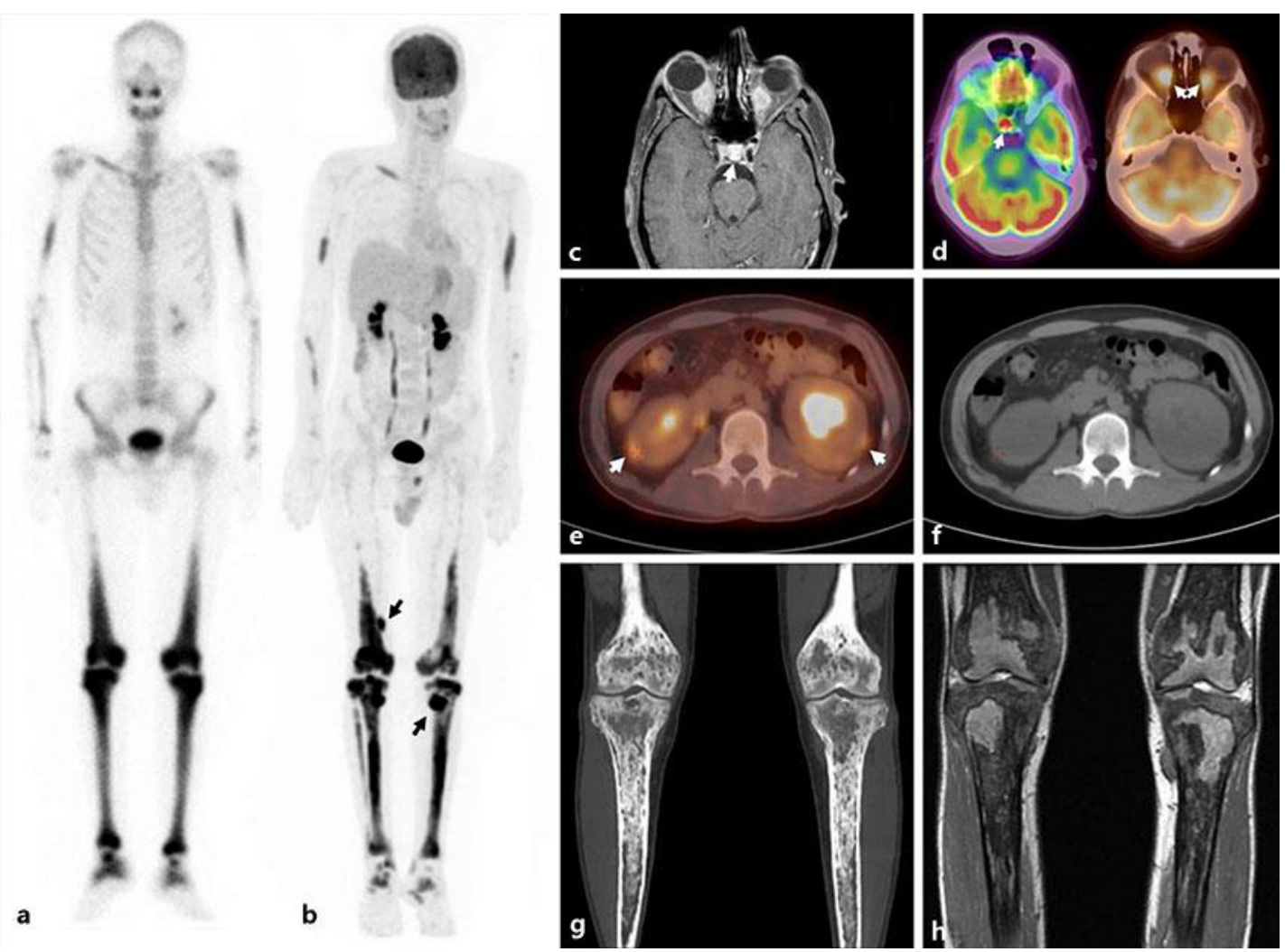

Fig. 2. ${ }^{99} \mathrm{mTc}$-methylene diphosphonate (a) and ${ }^{18 \mathrm{~F}}$-fluorodeoxyglucose positron emission tomography (FDG-PET) (b) show bilateral symmetric uptake in the long bones, right clavicle and mandible as well as subcutaneous and muscle soft tissue involvement in the lower limbs (black arrows). Computed tomography (CT) (g) and MRI (h) for the lower limbs revealed multifocal intramedullary sclerosis affecting both legs and distal portions of both femoral bones. Pituitary involvement and retrobulbar soft tissue infiltration were demonstrated as enhancing lesions on MRI (c) and high FDG uptake on PET/CT (d) brain images (white arrows). Fused PET/CT images and CT images (e, f) demonstrated bilateral perinephric soft tissue thickening and uptake (white arrows). 


\section{Case Reports in Oncology}

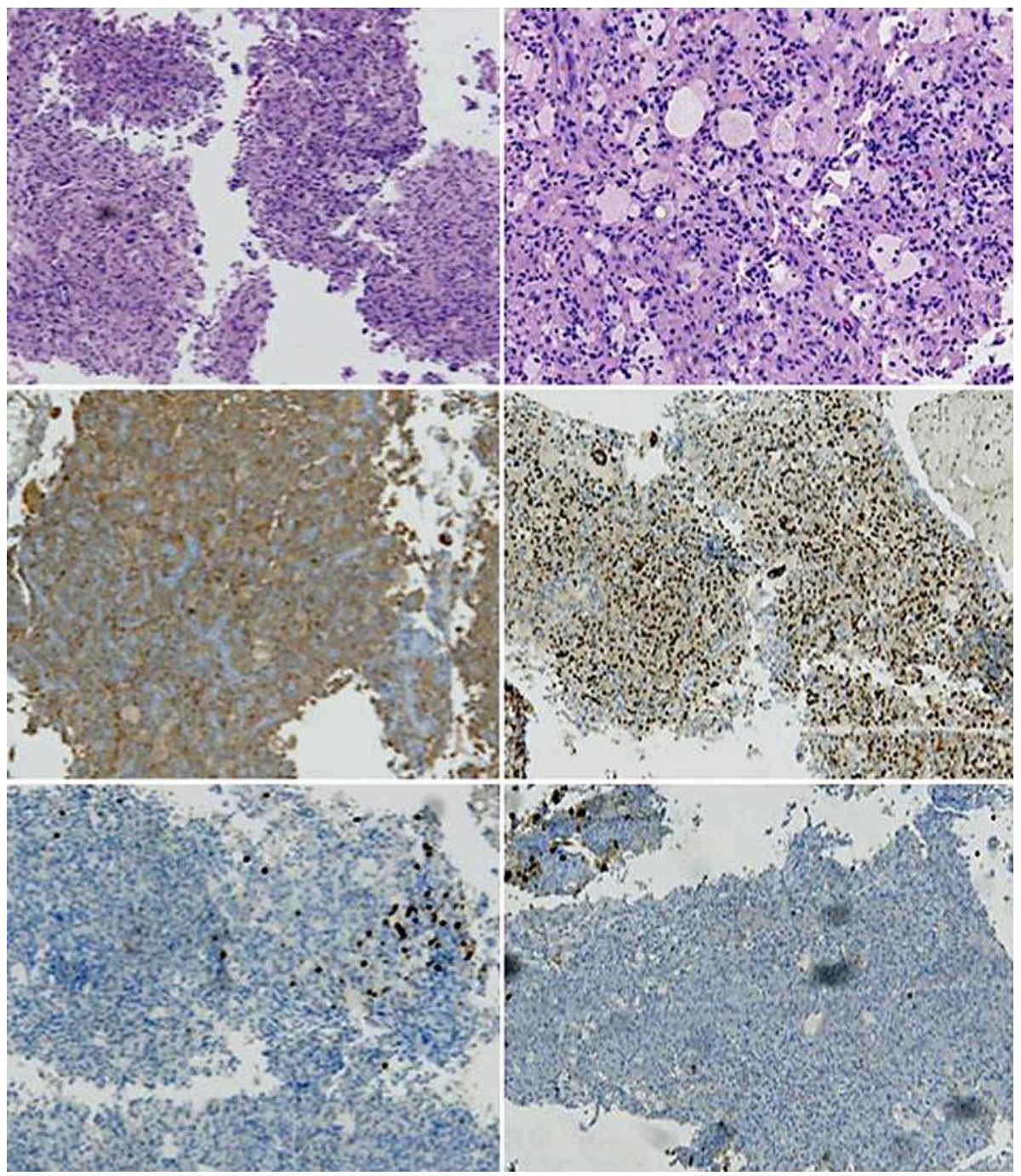

Fig. 3. Histopathologic findings of Erdheim-Chester disease. Low (left) and high power (right) of hematoxylin-eosin-stained biopsy section of Erdheim-Chester disease lesion revealing characteristic histiocytes, fibrosis, and multinucleated Touton giant cells. Immunohistochemistry studies revealing positivity of histiocytes to CD68-PMG1 and factor XIIIa, while negative to langerin and CD1a. 